\title{
Effects of compound Kushen injection on pathology and angiogenesis of tumor tissues
}

\author{
HONGYAN WANG ${ }^{1}$, HAIYAN HU ${ }^{2}$, HUA RONG ${ }^{3}$ and XUWEI ZHAO ${ }^{3}$ \\ ${ }^{1}$ Pharmacy of Traditional Chinese Medicine and ${ }^{2}$ Department of Gastroenterology, \\ The First Affiliated Hospital of Qiqihar Medical University; ${ }^{3}$ Institute of Pharmacology, \\ Qiqihar Medical University, Qiqihar, Heilongjiang 161000, P.R. China
}

Received April 30, 2018; Accepted November 7, 2018

DOI: $10.3892 / \mathrm{ol} .2018 .9861$

\begin{abstract}
Effects of compound Kushen injection on pathology and angiogenesis of tumor tissues were investigated. Forty nude mice were used to establish the liver cancer model of nude mice, and were divided into model group $(n=10)$, low-dose compound Kushen injection group $(n=10)$, medium-dose compound Kushen injection group $(n=10)$ and high-dose compound Kushen injection group $(n=10)$. When the tumor volume reached $0.5 \mathrm{~cm}^{3}, 200,400$ and $600 \mu 1$ of compound Kushen were injected into the mice of the low-, medium- and high-dose compound Kushen injection groups, respectively, for 3 consecutive days; while $400 \mu 1$ normal saline were injected into the mice of the model group. At 9 days after treatment, the mice were sacrificed, and the tumor was taken and weighed. The tumor inhibition rate was calculated, $\alpha$-smooth muscle actin ( $\alpha$-SMA) and cluster of differentiation 31 (CD31) were detected via immunohistochemistry, and the vascular maturity index (VMI) and microvessel density (MVD) were also detected. With the increase of compound Kushen injection dose, the tumor mass was decreased significantly $(\mathrm{P}<0.05)$, and the tumor inhibition rate was obviously increased $(\mathrm{P}<0.05)$. In the model group, the nuclei were large and deeply stained, and there were many mitotic figures, and more small blood vessels could be seen. In the three Kushen injection groups, the number of mitotic figures was slightly decreased, and the vascular distribution was reduced. With the increase of compound Kushen injection dose, MVD of transplanted tumor was decreased significantly, but VMI was increased significantly $(\mathrm{P}<0.05)$. Compound Kushen injection can reduce the angiogenesis in tumor tissues and play a role in
\end{abstract}

Correspondence to: Dr Hongyan Wang, Pharmacy of Traditional Chinese Medicine, The First Affiliated Hospital of Qiqihar Medical University, 26 Xiangyang Avenue, Qiqihar, Heilongjiang 161000, P.R. China

E-mail: hgo4rf@163.com

Key words: compound Kushen injection, liver cancer, anti-angiogenesis, vascular maturity index, microvessel density inhibiting tumor growth. Therefore, anti-angiogenesis may be one of the important mechanisms of compound Kushen injection in inhibiting tumor growth.

\section{Introduction}

Compound Kushen injection, made from Sophora flavescens and Rhizoma Heterosmilacis Japonicae, is a kind of commonly-used compound in traditional Chinese medicine prescriptions, and its active ingredients mainly include matrine, oxymatrine, saponin and oxysophocarpine, with effects of removing pathogenic heat from the blood and toxic material from the body, clearing heat and promoting diuresis, removing stasis and relieving pain (1). Current studies have shown that compound Kushen injection can affect the normal cycle of tumor cells or vascular endothelial growth factors (VEGFs), thus inducing cell apoptosis, inhibiting tumor cell proliferation and tumor metastasis, improving immune level in the body of cancer patients, and increasing physical condition and life quality (2). Therefore, compound Kushen injection has been widely used in the clinical treatment of tumors in China, such as ovarian, gastric, liver, breast and esophageal cancers $(3,4)$. However, the exact antitumor mechanism of compound Kushen injection remains unclear at present. This experiment aimed to further verify the antitumor effect of compound Kushen injection and reveal its mechanism in inhibiting tumor growth, so as to provide a scientific basis for its clinical application.

\section{Materials and methods}

Experimental animals and grouping. A total of 40 specific pathogen-free (SPF) BALB/cA-nu nude mice (20 male, 20 female), 4 weeks old, weighing 18-22 g, were purchased from the Laboratory Animal Center of Chinese Academy of Medical Sciences. The mice were kept in cages with controlled temperature, light cycles and humidity $\left(24^{\circ} \mathrm{C}\right.$ and $12 / 12$ light cycles, $60 \pm 10 \%$ ) and had free access to food and water. Mice were divided into model group ( $\mathrm{n}=10)$, low-dose compound Kushen injection group $(n=10)$, medium-dose compound Kushen injection group $(n=10)$ and high-dose compound Kushen injection group $(n=10)$ by using a random number table. The study was approved by the Ethics Committee of the First Affiliated Hospital of Qiqihar Medical University (Qiqihar, China). 
Main reagents. The following reagents were used: human hepatoma HepG2 cells (cat. no. CL-0103; Procell Life Science \& Technology Co., Ltd., Wuhan, China) (5), total ribonucleic acid (RNA) extraction kits (Shanghai Chaoyan Biotechnology Co., Ltd., Shanghai, China), rabbit anti-mouse $\alpha$-smooth muscle actin ( $\alpha$-SMA) and cluster of differentiation 31 (CD31) monoclonal antibodies (at dilution of 1:600 and 1:100, respectively; cat. nos. 19245 and 77699, respectively; both from Cell Signaling Technology, Inc., Danvers, MA, USA), Dulbecco's modified Eagle's medium (DMEM) and fetal bovine serum (FBS; both from Gibco; Thermo Fisher Scientific, Inc., Waltham, MA, USA). The rest of the reagents were analytically pure reagents made in China.

Main instruments. The following instruments were used: ultra-clean bench (Air Tech, Suzhou Purification Equipment Co., Ltd., Suzhou, China), precision electronic balance (Adventurer Precision; OHAUS Corp., Parsippany, NJ, USA), upright fluorescence microscope (Olympus Corp., Tokyo, Japan), reverse transcription-polymerase chain reaction (RT-PCR) instrument (Biometra, Göttingen, Germany), dual-vertical protein electrophoresis apparatus (Beijing Liuyi Instrument Factory, Beijing, China), ultraviolet spectrophotometer (NanoDrop; Thermo Fisher Scientific, Inc., Waltham, MA, USA), and microplate reader (Thermo Fisher Scientific, Inc.).

Establishment of liver cancer model of nude mice and treatment. HepG2 cells in the logarithmic growth phase were collected, washed twice with phosphate-buffered saline (PBS), stained with trypan blue and counted. After the cell concentration was adjusted to $1 \times 10^{7} / \mathrm{ml}, 0.2 \mathrm{ml}\left(2 \times 10^{6}\right.$ cells $)$ of cell suspension were taken by using a syringe, and inoculated into subcutaneous tissues on the back of nude mice. The life changes and tumor growth were observed every day; the long diameter (L) and short diameter (W) of tumor were measured every 3 days, and the tumor volume was calculated according to the formula $\mathrm{V}=\mathrm{L}_{\mathrm{x} \mathrm{W}} \times \mathrm{W}^{2} \mathrm{x} 0.52$. When the tumor volume reached $0.5 \mathrm{~cm}^{3}$ (the baseline level before treatment), 200, 400 and $600 \mu \mathrm{l}$ of compound Kushen were injected into the mice of the low-dose, medium-dose and high-dose compound Kushen injection groups, respectively, for 3 consecutive days; while $400 \mu \mathrm{l}$ normal saline was injected into the mice of the model group. There were no significant differences in body weight, tumor volume and time of subcutaneous cell inoculation among groups of mice. At 9 days after treatment, the mice of each group were executed, and the tumors were taken and weighed. The tumor inhibition rate was calculated; $\alpha$-SMA and CD31 were detected via immunohistochemistry, and microvessel density (MVD) and vascular maturity index (VMI) were also detected.

Tumor inhibition rate. The tumor inhibition rate was calculated using the formula: Tumor inhibition rate $(\%)=$ (tumor weight $_{\text {model }}$ group - tumor weight drug administration group $)$ /tumor weight model group .

Immunohistochemical detection. After computed tomography (CT) scan, 5 mice of each group were sacrificed, and tumors were removed and embedded in paraffin. MVD and $\alpha$-SMA expression were detected via immunohistochemical
Table I. Comparison of tumor inhibition rate among groups.

\begin{tabular}{lccc}
\hline Group & $\mathrm{n}$ & $\begin{array}{c}\text { Tumor } \\
\text { mass }(\mathrm{g})\end{array}$ & $\begin{array}{c}\text { Tumor } \\
\text { inhibition } \\
\text { rate }(\%)\end{array}$ \\
\hline $\begin{array}{l}\text { Model group } \\
\text { Low-dose compound }\end{array}$ & 10 & $1.65 \pm 0.44$ & \\
$\begin{array}{l}\text { Kushen injection group } \\
\text { Medium-dose compound } \\
\text { Kushen injection group }\end{array}$ & 10 & $1.41 \pm 0.21^{\mathrm{a}}$ & 14.55 \\
$\begin{array}{l}\text { High-dose compound } \\
\text { Kushen injection group }\end{array}$ & 10 & $1.06 \pm 0.15^{\mathrm{a}, \mathrm{b}}$ & $24.24^{\mathrm{a}-\mathrm{c}}$ \\
\hline
\end{tabular}

${ }^{\mathrm{a}} \mathrm{P}<0.05$ vs. model group, ${ }^{\mathrm{b}} \mathrm{P}<0.05$ vs. low-dose compound Kushen injection group, ${ }^{\mathrm{C}} \mathrm{P}<0.05$ vs. medium-dose compound Kushen injection group.

staining, and they were labeled with CD31 and $\alpha$-SMA antibodies, respectively. Specific operations are as follows: $\alpha$-SMA was incubated with primary and secondary antibodies, dropwise added with QDs-IgG complex (labeling CD31) and QDs- $\alpha$-SMA complex (labeling $\alpha$-SMA), and were observed under an upright fluorescence microscope (Nikon Instruments, Inc., Melville, NY, USA).

MVD detection of transplanted tumor. After 3 fields of view were randomly selected under high-power lens (x200), the red CD31 fluorescence signal (CD31-positive marker showing red fluorescence) was used to label mature and immature vessels. MVD criteria: CD31-positive endothelial cells showing red fluorescence were clearly separated from tumor cells, adjacent blood vessels and other connective tissues. The average microvessel count was taken, and microvessels in tumor-adjacent normal structure were not counted.

VMI detection of transplanted tumor. In the same field of view in MVD detection, ImageJ professional image analysis software (National Institutes of Health, Bethesda, MD, USA) was used to quantify the expression levels of CD31 and VEGF, and VMI was calculated $(\mathrm{VMI}=\alpha-\mathrm{SMA}$ expression level/CD31 expression level).

Statistical analysis. Statistical Product and Service Solutions (SPSS) 18.0 software (SPSS, Inc., Chicago, IL, USA) was used for the statistical analysis of all data. t-test was used for measurement data and the results were presented as mean \pm standard deviation (SD). ANOVA and LSD test were used for the comparison of multiple groups. $\mathrm{P}<0.05$ was considered to indicate a statistically significant difference.

\section{Results}

Tumor inhibition rate. Compared with that in the model group, the tumor weight in low-dose, medium-dose and high-dose compound Kushen injection groups was significantly decreased $(\mathrm{P}<0.05)$. With the increase of compound Kushen injection dose, the tumor mass was decreased significantly 

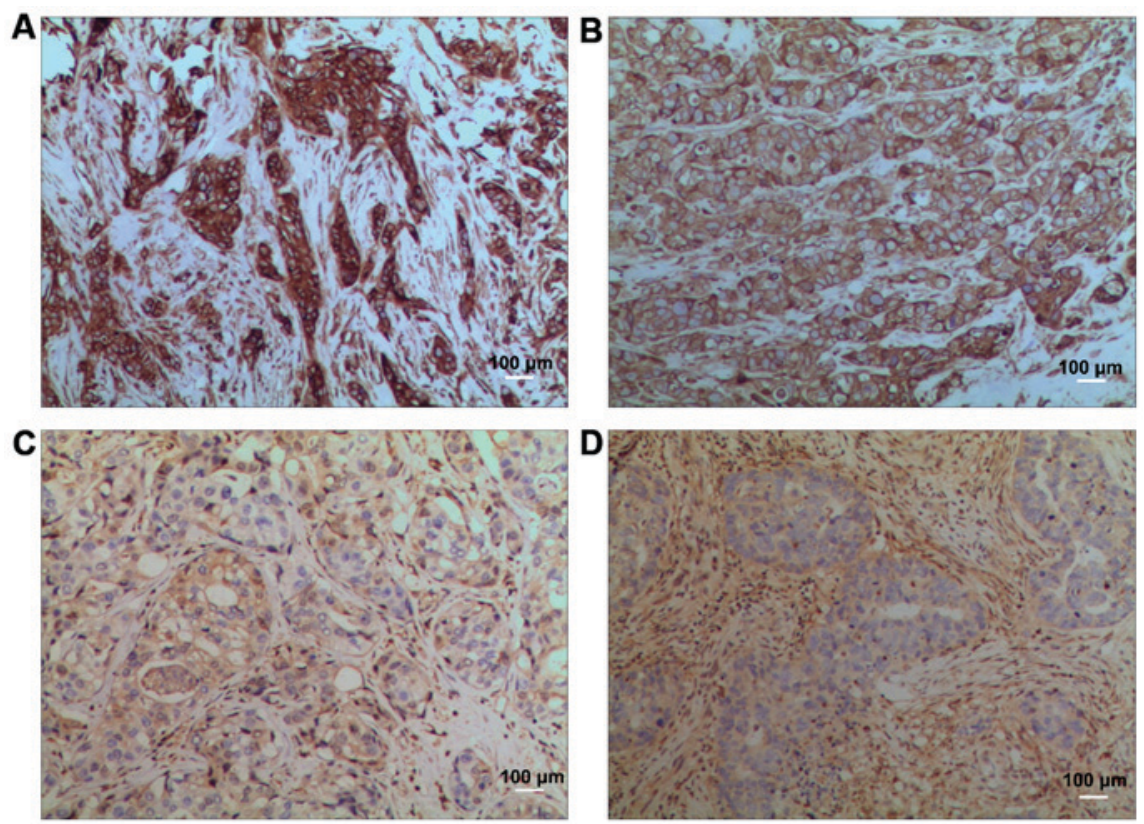

Figure 1. Hematoxylin and eosin (H\&E) staining results. (A) Model group, (B) low-dose compound Kushen injection group, (C) medium-dose compound Kushen injection group, (D) high-dose compound Kushen injection group.

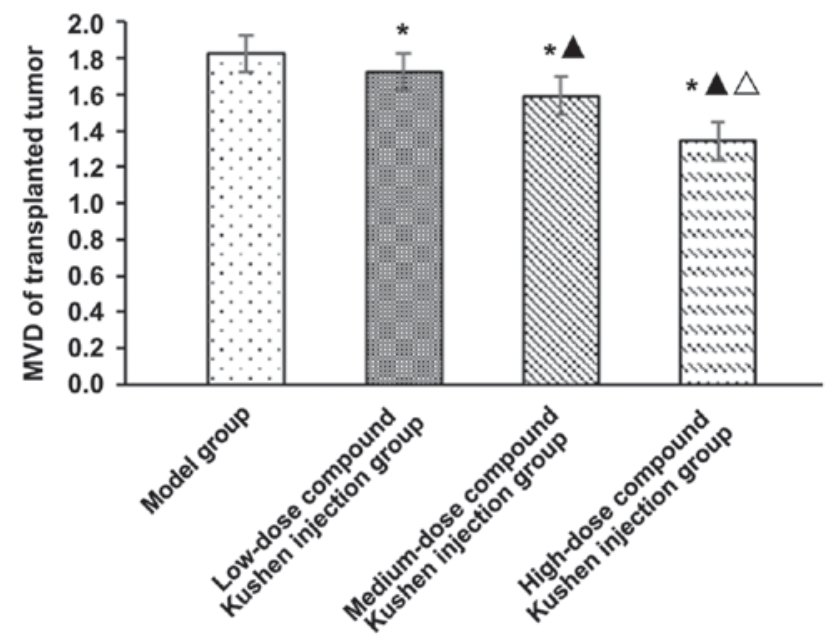

Figure 2. Effect of different doses of compound Kushen injection on MVD of transplanted tumor in mice. ${ }^{*} \mathrm{P}<0.05$ vs. model group, ${ }^{\wedge} \mathrm{P}<0.05$ vs. low-dose compound Kushen injection group, ${ }^{\triangle} \mathrm{P}<0.05$ vs. medium-dose compound Kushen injection group. MVD, microvessel density.

$(\mathrm{P}<0.05)$, and the tumor inhibition rate was obviously increased $(\mathrm{P}<0.05)$ (Table I).

Morphological changes. In model group, the nuclei were large and deeply stained, there were many mitotic figures, and more small blood vessels could be seen. In low-dose compound Kushen injection group, the number of mitotic figures was slightly decreased, and the vascular distribution was also reduced. In medium-dose compound Kushen injection group, the number of mitotic figures was decreased, and the vascular distribution was also significantly reduced. In high-dose compound Kushen injection group, the number of mitotic figures was decreased, and the vascular distribution was further reduced (Fig. 1).

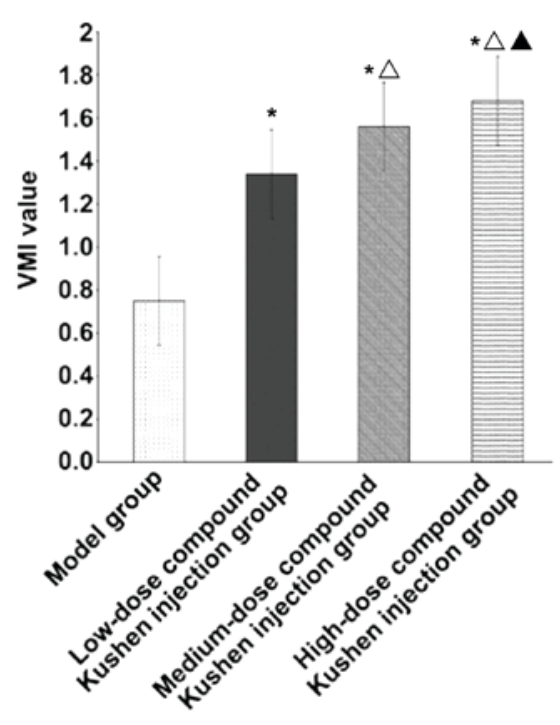

Figure 3. Effect of different doses of compound Kushen injection on VMI of transplanted tumor in mice. ${ }^{~} \mathrm{P}<0.05$ vs. model group, ${ }^{\mathrm{A}} \mathrm{P}<0.05$ vs. low-dose compound Kushen injection group, ${ }^{\triangle} \mathrm{P}<0.05$ vs. medium-dose compound Kushen injection group. VMI, vascular maturity index.

MVD of transplanted tumor. Compared with that in the model group, MVD of transplanted tumor in low-, medium- and high-dose compound Kushen injection groups was obviously decreased $(\mathrm{P}<0.05)$. With the increase of compound Kushen injection dose, MVD of transplanted tumor was decreased significantly $(\mathrm{P}<0.05)$ (Fig. 2).

VMI of transplanted tumor. Compared with that in the model group, VMI of transplanted tumor in low-dose, medium-dose and high-dose compound Kushen injection groups was obviously increased $(\mathrm{P}<0.05)$. With the increase of compound Kushen injection dose, VMI of transplanted tumor was increased significantly $(\mathrm{P}<0.05)$ (Fig. 3). 


\section{Discussion}

Compound Kushen injection has been widely used in the treatment of cardiovascular and rheumatic diseases as well as viral hepatitis (2). In addition, compound Kushen injection has a broad-spectrum of antitumor effects, which can not only enhance antitumor and chemoradiotherapy sensitization, and reduce relevant side-effects of chemoradiotherapy, but also exert effects of hemostasis, analgesia and regulation of the body immunity (6). In this study, the effects of compound Kushen injection on pathology and angiogenesis of tumor tissues in mice with liver cancer were analyzed, so as to clarify the antitumor mechanism of compound Kushen injection.

Results of this study showed that with the increase of compound Kushen injection dose, the tumor mass was significantly decreased $(\mathrm{P}<0.05)$, and the tumor inhibition rate was significantly increased $(\mathrm{P}<0.05)$, indicating that compound Kushen injection can significantly inhibit the growth of transplanted tumor in nude mice in a dose-dependent manner. Compound Kushen injection is extracted and prepared by two medicinal materials: Sophora flavescens and Rhizoma Heterosmilacis Japonicae. Oxymatrine (molecular formula: $\mathrm{C}_{15} \mathrm{H}_{24} \mathrm{~N}_{2} \mathrm{O}_{2}$ ) is one of the main active ingredients of Sophora flavescens, and it belongs to quinolizidine alkaloid, which is colorless crystal and easily soluble in water with relative molecular mass of 264.4 (7). Modern pharmacological studies have shown that oxymatrine has a wide range of pharmacological effects, such as antivirus, antitumor, anti-hypertension, heart strengthening, antipyresis and analgesia, inhibition of myocardial ischemia and infarction, asthma relieving, anti-arrhythmia, sterilization, anti-inflammation, sedation and hypnosis, and anti-allergy $(8,9)$. In Rhizoma Heterosmilacis Japonicae, flavonoids and triterpenes have extensive anti-inflammatory, detoxification and antitumor effects (10).

Anti-angiogenic therapy is a therapeutic method to prevent and/or reduce angiogenesis in diseased tissues. Currently, commonly-used anti-angiogenic drugs in clinic, such as bevacizumab, recombinant human endostatin injection and imatinib, have achieved varying degrees of effects, bringing new hope for the clinical treatment of tumors (11). Studies have shown that anti-angiogenic therapy combined with surgery, thermal therapy and chemoradiotherapy is more conducive to improving the curative effect (12). The results of this study showed that with the increase of compound Kushen injection dose, the mitotic figure of tumor cells and vascular distribution in tumor tissues is reduced, thereby inhibiting tumor cell division and proliferation, suggesting that compound Kushen injection may play an antitumor effect through anti-angiogenesis.

Further analysis showed that with the increase of compound Kushen injection dose, MVD of transplanted tumor is significantly decreased $(\mathrm{P}<0.05)$, but VMI was significantly increased $(\mathrm{P}<0.05)$. MVD can reflect the rates of changes in tumor cell components and tumor vascular components, while VMI can quantitatively analyze the changes in vascular maturity and is more representative in the microvascular functional status than MVD (13,14). Therefore, compound Kushen injection may inhibit tumor angiogenesis to reduce tumor blood supply, thereby inhibiting tumor growth. Some studies have shown that compound Kushen injection can reduce the expression of
VEGF. VEGF, as an important regulator of angiogenesis, plays an important role in regulating neovascularization in endothelial cells in tumor blood vessels, so when its level is reduced, angiogenesis is limited, and tumor blood supply is reduced, thus inhibiting tumor growth (15).

In conclusion, compound Kushen injection can reduce angiogenesis in tumor tissues and plays a key role in inhibiting tumor growth. Therefore, anti-angiogenesis may be one of the important mechanisms of compound Kushen injection in inhibiting tumor growth.

\section{Acknowledgements}

Not applicable.

\section{Funding}

This study was supported by the mandatory scientific research of Qiqihar Science and Technology Bureau (SFGG-201415).

\section{Availability of data and materials}

The datasets used and/or analyzed during the current study are available from the corresponding author on reasonable request.

\section{Authors' contributions}

HW and HH contributed to the establishment of the liver cancer model. HR was responsible for the immunohistochemical detection. XZ assisted with MVD detection. All authors read and approved the final manuscript.

\section{Ethics approval and consent to participate}

This study was approved by the Ethics Committee of the First Affiliated Hospital of Qiqihar Medical University (Qiqihar, China).

\section{Patient consent for publication}

Not applicable.

\section{Competing interests}

The authors declare that they have no competing interests.

\section{References}

1. Yu L, Zhou Y, Yang Y, Lu F and Fan Y: Efficacy and safety of Compound Kushen Injection on patients with advanced colon cancer: A meta-analysis of randomized controlled trials. Evid Based Complement Alternat Med 2017: 7102514, 2017.

2. Wang W, You RL, Qin WJ, Hai LN, Fang MJ, Huang GH, Kang RX, Li MH, Qiao YF, Li JW, et al: Anti-tumor activities of active ingredients in Compound Kushen injection. Acta Pharmacol Sin 36: 676-679, 2015.

3. Li J, Wu M, Tian Q, Xie G, Hu Y, Meng Q and Zhang M: The clinical value of Fufangkushen injection in the treatment of stomach cancer: A meta-analysis. J Cancer Res Ther 10 (Suppl 1): 42-45, 2014.

4. Yanju B, Yang L, Hua B, Hou W, Shi Z, Li W, Li C, Chen C, Liu R, Qin Y, et al: A systematic review and meta-analysis on the use of traditional Chinese medicine compound Kushen injection for bone cancer pain. Support Care Cancer 22: 825-836, 2014. 
5. López-Terrada D, Cheung SW, Finegold MJ and Knowles BB Hep G2 is a hepatoblastoma-derived cell line. Hum Pathol 40: $1512-1515,2009$.

6. Zhao Z, Liao H and Ju Y: Effect of compound Kushen injection on T-cell subgroups and natural killer cells in patients with locally advanced non-small-cell lung cancer treated with concomitant radiochemotherapy. J Tradit Chin Med 36: 14-18, 2016.

7. Cao YG, Jing S, Li L, Gao JQ, Shen ZY, Liu Y, Xing Y, Wu ML, Wang Y, Xu CQ, et al: Antiarrhythmic effects and ionic mechanisms of oxymatrine from Sophora flavescens. Phytother Res 24: 1844-1849, 2010

8. Wang S, Lian X, Sun M, Luo L and Guo L: Efficacy of compound Kushen injection plus radiotherapy on non-small cell lung cancer: A systematic review and meta-analysis. J Cancer Res Ther 12: 1298-1306, 2016.

9. Malhotra J, Jabbour SK and Aisner J: Erratum to current state of immunotherapy for non-small cell lung cancer. Transl Lung Cancer Res 6: 612, 2017.

10. Guo YM, Huang YX, Shen HH, Sang XX, Ma X, Zhao YL and Xiao XH: Efficacy of Compound Kushen injection in relieving cancer-related pain: A systematic review and meta-analysis. Evid Based Complement Alternat Med 2015: 840742, 2015.

11. Olaso E and Vidal-Vanaclocha F: Use of tumor-activated hepatic stellate cell as a target for the preclinical testing of anti-angiogenic drugs against hepatic tumor development. Methods Mol Med 85 : 79-86, 2003.
12. Browder T, Butterfield CE, Kräling BM, Shi B, Marshall B, O'Reilly MS and Folkman J: Antiangiogenic scheduling of chemotherapy improves efficacy against experimental drug-resistant cancer. Cancer Res 60: 1878-1886, 2000.

13. Zhao Z, Fan H, Higgins T, Qi J, Haines D, Trivett A, Oppenheim JJ, Wei H, Li J, Lin H, et al: Fufang Kushen injection inhibits sarcoma growth and tumor-induced hyperalgesia via TRPV1 signaling pathways. Cancer Lett 355: 232-241, 2014.

14. Lee CN, Cheng WF, Chen CA, Chu JS, Hsieh CY and Hsieh FJ: Angiogenesis of endometrial carcinomas assessed by measurement of intratumoral blood flow, microvessel density, and vascular endothelial growth factor levels. Obstet Gynecol 96: 615-621, 2000

15. Carmeliet P: VEGF as a key mediator of angiogenesis in cancer. Oncology 69 (Suppl 3): 4-10, 2005.

(i) (3) This work is licensed under a Creative Commons

EY NO NO Attribution-NonCommercial-NoDerivatives 4.0 International (CC BY-NC-ND 4.0) License. 